\title{
Spatial Motion of Multi-Pendula Systems
}

\author{
J. S. Prichani ${ }^{1}$, T. W. Sakwa ${ }^{2}$, N. O. Ongati ${ }^{3}$ \\ ${ }^{1}$ Masinde Muliro University of Science and Technology, Mathematics Department, P.o Box 1388-50205, Webuye, Kenya \\ ${ }^{2}$ Masinde Muliro University of Science and Technology, Mathematics Department, P.o Box 190-50100, Kakamega, Kenya \\ ${ }^{3}$ Jaramogi Oginga Odinga University Science and Technology, Department of Mathematics, P.o. Box 210-40601, Bondo, Kenya
}

\begin{abstract}
A multiple chain pendula system constrained to move in space has been studied within the framework of a generalized coordinate system by using the Lagrangian formalism. Equations of motions for many body pendula systems have been derived .These equations concur very well with known data. We confirm that equations of motion for any values of $n$ and $l$ can be generated from our general equation which presents interesting characteristics. Solutions to these multi-pendula equations could be deduced.
\end{abstract}

Keywords: Multi-pendula system, Multi-pendulum systems, Zenith, Azimuth and Angular accelerations

\section{Introduction}

The study of the equations of motion for multi-pendula systems has been widely explo Braun (2002) acknowledged that a multiple pendulum was considered for a plane motion in a vertical plane only and to establish the equations for $n$ $>1$. These solutions were found by Prichani et al (2010) for the n-tupple pendulum systems. They used the Lagrangian framework. In this it was observed that the angular acceleration for any mass was influenced by the immediate masses and angles.

Moore et al(1990) did the experimental response of an impacting pendulum system. Goriety et al (2006) researched on models about the jumping pendulum. They studied conditions to attain the jump position and move as a projectile. McMillen et al (2003) researched on the whipwaves which resulted from the sudden impulse shock on the red pendulum. A comparison of the velocity and the time scale associated with the periodic motion of the pendulum gave a conclusion that the time required for the completion of the energy loss related to the shock was negligible.

Joot (2009) wrote about the multi- spherical pendula system with $\mathrm{n}$ components where energy was described and acknowledged that the description of the dynamics of the multi- pendula was of formidable difficulty. They therefore concentrated on the more accessible problem of small oscillations.

Martin and Egerstedt (2007) had made efforts to develop robotic marionettes. They switched systems with robotic marionettes in a workshop on Discrete Event systems. Maziar and Andre (2015) in Gemany studied about the leg function and the ground reaction force in legged locomotion. They used the leg force modulated complaint hip in a new model for postural control in walking which employs the leg force feedback to adjust the hip compliance. This method gives a stable and robust walking in simulations and imitates human-like kinetic behavior. This gives the hip torque-angle relation for different walking speeds. The approach may physically implement the virtual pendulum concept in human animal locomotion. Tri-Nhut et al (2015) considered the demand for firemen and soldiers that could not be gainsaid for the need of safety and strategy in planning and co-ordination. In their paper, it is presented a new method to estimate both the forward displacement and orientation. The sensor unit is placed at the pedestrians ankles for the greater ease of usage. The 2D displacement is then computed based on the estimations for the pitch angle, yaw angle and the pedestrians leg length. This leg length is automatically estimated during the walking by exploiting the motion equation of a simple pendulum model and hence no prior measurements or training is required. This method employs the quaternion-based indirect kalman filter to estimate the Euler angles containing the yaw angle, the pitch angle and the roll angle. The real-time localization system has been implemented and experiments for various subjects conducted. The results demonstrated the accuracy with the average displacement error of less than 3\%. Leonard and Seth (2015) considered the problem of simultaneously synthesizing desired trajectories for both the centre of mass and the zero moment point of a bipedal robot. They parameterized the desired zero moment point trajectories by a sequence of basic functions where the design parameters were optimized by an algorithm in which the centre of mass boundedness constraint as well as the zero moment point and other centre of mass are simultaneously satisfied.

Martin et al (2015) used an inverted pendulum to calculate the length of gait in human locomotion. This is approximated by a simple pendulum where the influence of the knee and the ankle movements are not involved. The gait length is calculated from the extremes of vertical position of the human centre of mass during gait. The position of the centre of mass is calculated by double integration of acceleration which has errors. This article shows the influence of the inverted pendulum model as a methodology to measure the length of gait. Zhenglong and Nico (2014) in their paper presented a framework for energy efficient dynamic human-like walk for a Nao humanoid robot. They used an inverted pendulum model to find an energy efficient stable walking gait. In this model they proposed a leg control policy which utilizes joint stiffness control. In order to identify the optimal parameters of the new gait for a Nao humanoid robot they used the policy gradient reinforcement. On testing this policy in a simulator and on a real Nao robot it was successful. It was shown that the new control policy 


\section{International Journal of Science and Research (IJSR) \\ ISSN (Online): 2319-7064}

Index Copernicus Value (2015): 78.96 | Impact Factor (2015): 6.391

had a dynamic walk that is more energy efficient than the standard walk of a Nao robot.

According to a paper posted by Joot (2009) it was indicated that although setting up the Lagrangian, for a double pendulum was difficult, it was worse solving it. From his research he did not know what to expect in future. He avoided doing more work because it proved to be complicated. In his second paper posted by the said Joot (2010), it was noted that introducing any additional mass in the system, for planer motion, the interaction coupling terms increase and thus complicate the kinetic energy specifications. This concept is captured by Prichani et al (2012) where it was stated that if there are $n$ masses suspended there will be $\frac{n(n+1)}{2}$ kinetic energy terms and for $(n+1)$ mass units, these terms will be $\frac{(n+1)(n+2)}{2}$.It was observed that an increase by one mass would give an increase of $(n+1)$ kinetic energy terms.

In this study we considered a freely moving system whose mass displacements are very small with different mass sizes, varying inter-link lengths with masses at different angular displacements at different times. A rather comprehensive study and contributions on systems of multiple masses suspended and connected by light inextensible pieces of thread at small angles of inclination to the vertical, resolved in the horizontal and vertical positions, by using the Lagrange method has been done. We have been specific in varying masses and lengths and noted that by introducing additional mass units in the system, the interacting coupling terms increase thus complicating the kinetic energy specifications

Calculation of the energy explicitly for a general n-pendula system is derived. We have restricted our work to a series of n-pendula systems oscillating in space. This finds interesting applications in the study various systems like describing the spin and orbital dynamics of super fluids.

\section{The Energy Equation}

The Lagrangian formulation for suspended masses $m_{i}$ at distances of separation $l_{i}$ and at angles of inclination $\theta_{i}$ and $\phi_{i}$, to the vertical and horizontal axes respectively, is first developed. The general resultant velocity component is calculated from the component of the effective displacements as follows:

$$
\begin{gathered}
\dot{x}_{k}=\sum_{i=1}^{k} l_{i}\left(\dot{\theta}_{i} \cos \theta_{i} \cos \emptyset_{i}-\dot{\emptyset}_{i} \sin \theta_{i} \sin \emptyset_{i}\right) \\
\dot{y}_{\boldsymbol{k}}=\sum_{i=1}^{k} l_{i}\left(\dot{\theta}_{i} \cos \theta_{i} \sin \emptyset_{i}+\dot{\emptyset}_{i} \sin \theta_{i} \cos \emptyset_{i}\right) \\
\dot{z}_{\boldsymbol{k}}=\sum_{i=1}^{k}\left(-l_{i} \dot{\theta}_{i} \sin \theta_{i}\right)
\end{gathered}
$$

$$
\begin{gathered}
\dot{r}_{k}^{2}=\dot{x}_{k}^{2}+\dot{y}_{k}^{2}+\dot{z}_{k}^{2}=\sum_{i=1}^{2} \theta_{i}^{2}\left(\theta_{i}^{2}+\dot{\Phi}_{i}^{2} \sin ^{2} \theta_{i}\right)+2 \sum_{\substack{i=1 \\
i \neq j}}^{k} l_{i} l_{j}\left\{\theta_{i} \theta_{j} \cos \left(\theta_{i}-\theta_{j}\right)+\dot{\Phi}_{i} \dot{\phi}_{j} \sin \theta_{i} \sin \theta_{j}\right\} \\
\text { The total kinetic energy T is established by taking a summation over all values of k. } \\
T=\sum_{k=1}^{n} m_{k}\left[\frac{1}{2} \sum_{i=1}^{n} l_{i}^{2}\left(\dot{\theta}_{i}^{2}+\dot{\emptyset}_{i}^{2}\right)+\sum_{\substack{i=1 \\
i \neq j}}^{n} l_{i} l_{j}\left\{\left(\dot{\theta}_{i} \dot{\theta}_{j} \cos \left(\theta_{i}-\theta_{j}\right)+\dot{\emptyset}_{i} \dot{\phi}_{j} \sin \theta_{i} \sin \theta_{j}\right)\right\}\right]
\end{gathered}
$$

Similarly the total potential energy is calculated as

$$
\boldsymbol{V}=\sum_{k=1}^{n} m_{k}\left(\sum_{i=1}^{n} l_{i}-\sum_{i=1}^{n} l_{i} \cos \theta_{i}\right)
$$

From equations (5) and (6) we develop the Lagrangian for the multi-pendula system as

$$
\begin{gathered}
\mathbf{L}=\sum_{k=f(i, j)}^{n} m_{k}\left\{\sum_{i=1}^{n} \frac{I_{1}^{2}}{2}\left[\tilde{\theta}_{i}^{2}+\dot{\phi}_{i}^{2} \sin ^{2} \theta_{i}\right]+\sum_{i \neq j}^{n} l_{i} l_{j}\left[\tilde{\theta}_{i} \dot{\theta}_{j} \cos \left(\theta_{i}-\theta_{j}\right)+\dot{\emptyset}_{i} \dot{\phi}_{j} \sin \theta_{i} \sin \theta_{j}\right]\right\}-\sum_{k=1}^{n} m_{k}\left\{\left(\sum_{i=1}^{n} l_{i}\right)-\sum_{k=1}^{n} l_{i} \cos \theta_{i}\right\} g \\
\text { Then } \frac{\partial L}{\partial \dot{\phi}_{i}}=\sum_{k=i}^{n} m_{k}\left(l_{i}^{2} \dot{\phi}_{i} \sin ^{2} \theta_{i}\right)+\sum_{k=f(i j)}^{n} m_{k} l_{i} \sum_{i=1}^{n}\left(l_{j} \dot{\phi}_{j} \sin \theta_{i} \sin \theta_{j}\right)
\end{gathered}
$$

Differentiating this equation with respect to time yields

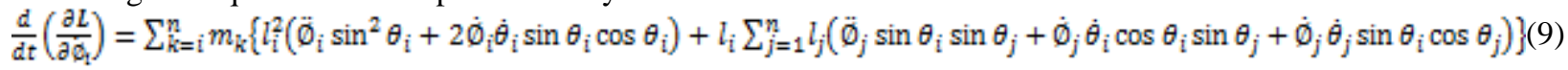

When

$\theta_{(i, j)} \cong 0 \frac{d}{d t}\left(\frac{\partial L}{\partial \dot{\emptyset}_{i}}\right)=\sum_{k=i}^{n} m_{k}\left\{l_{i}^{2}\left(\ddot{\emptyset}_{i} \theta_{i}^{2}+2 \dot{\emptyset}_{i} \dot{\theta}_{i} \theta_{i}\right)\right\}+\sum_{k=f(i, j)}^{n} m_{k}\left\{l_{i} \sum_{j=1}^{n} l_{j}\left(\ddot{\emptyset}_{j} \theta_{i} \theta_{j}+\dot{\emptyset}_{j} \dot{\theta}_{i} \theta_{j}+\dot{\emptyset}_{j} \dot{\theta}_{j} \theta_{i}\right)\right\}$

Also

$$
\frac{\partial L}{\partial \phi_{i}}=0
$$

For the multi-pendula systems we use the generalized energy equation to generate the azimuth angular acceleration equations

$$
\begin{aligned}
& \ddot{\emptyset}_{1}=\frac{1}{l_{1} m_{1}}\left\{\frac{-2 c_{1}}{\theta_{1}^{\mathrm{a}}}+\frac{c_{2}\left(\theta_{1} \theta_{2}+\theta_{1} \theta_{2}\right)}{\theta_{1}^{\mathrm{a}} \theta_{2}^{\mathrm{z}}}\right\} \\
& =\frac{1}{l_{1} m_{1}}\left\{\frac{-2 c_{1}}{\theta_{1}^{\mathrm{g}}}+c_{2}\left(\frac{\theta_{1}}{\theta_{1}^{\mathrm{a}} \theta_{2}}+\frac{\theta_{2}}{\theta_{1} \theta_{2}^{\mathrm{a}}}\right)\right\}
\end{aligned}
$$

Volume 6 Issue 7, July 2017 www.ijsr.net 


\section{International Journal of Science and Research (IJSR)}

ISSN (Online): 2319-7064

Index Copernicus Value (2015): 78.96 | Impact Factor (2015): 6.391

$$
\begin{aligned}
\ddot{\emptyset}_{(n-1)} & =\frac{1}{l m}\left\{\left(\frac{\theta_{(n-1)}}{\theta_{(n-1)}^{2} \theta_{(n-2)}}+\frac{\theta_{(n-2)}}{\theta_{(n-1)} \theta_{(n-2)}^{2}}\right) c_{(n-2)}-\frac{4 c_{(n-1)}}{\theta_{(n-1)}^{s}}+\left(\frac{\theta_{(n-1)}}{\theta_{(n-1)}^{2} \theta_{n}}+\frac{\theta_{n}}{\theta_{(n-1)} \theta_{n}^{2}}\right) c_{n}\right\} \\
\ddot{\emptyset}_{n} & =\frac{1}{l m}\left\{\left(\frac{\theta_{(n-1)}}{\theta_{(n-1)}^{2} \theta_{n}}+\frac{\theta_{n}}{\theta_{(n-1)} \theta_{n}^{2}}\right) c_{(n-1)}-\frac{4 c_{n}}{\theta_{n}^{g}}\right\}
\end{aligned}
$$

The azimuth angular accelerations $\ddot{\emptyset}_{1}, \ddot{\emptyset}_{(n-1)}$ and $\ddot{\emptyset}_{n}$ are given by equations (12), (13), (14) and (15) respectively. It was observed that these accelerations are directly proportional to the zenith angular velocity but vary inversely as the zenith angular displacements.

\section{Conclusion}

The objectives outlined above have been achieved. In general this research has developed the governing relations of spatial motion for the multiple pendula system set to oscillate in space for very small angular displacements. The Lagrangian formulation to the system in spatial dynamics has been established. The energy equations in spatial multiple pendula dynamical system have been deduced.

Since this work is restricted to motion in space for the Lagrangian formulation,one may want to compae with the Hamiltonian formulations for $\mathrm{n}$ suspended mass units. Engineering research could be carried out to determine the practicability of these results. The achievements may be useful to robotic engineering, theoretical physics and mechanics.

\section{Acknowledgements}

The authors are very grateful to Jaramogi Oginga Odinga University of Science and Technology for availing the necessary materials and facilities for the preparation of this manuscript.

\section{References}

[1] Braun M. (2002), properties of Multiple Pendulum, Institute for Mechanic and System Dynamics, Gerherd - Mercator University Dulsburg, 47048 Dulsburg, Germany.

[2] Goriely A., Philippe B. and Jules L., (2006) "Toy models: The jumping pendulum," American Journal of physics $74,784-788$.

[3] Joot P B,(2009),Spherical polar pendulum for one and multiple masses. RCs file:multipendulum spherical 2.tex, v last Revision: 1.20.

[4] Joot P.B., (2009), A Paper on Multiple pendulums.

[5] Joot P.B., (2010), A Paper on Hamiltonian for pendulum systems.

[6] Leonard L. and Seth H. (2015). Planning desired centre of mass and zero moment Point trajectories for bipedal motion.Humanoid Robots (Humanoids). ISBN 15680065.DOI: 10.1109/HUMAOIDS.2015.7363438.

[7] Martin C., Nobert N., Ludovic D., (2015)x Validation of inverted pendulum model for gait length calculation.Applied Machine intelligence and informatics (SAMI).ISBN1499924.
[8] Martin P. and Egerstedt M., (2007).Switched system with Application to robotic marionettes. In Workshop a Discrete Event systems Gotheriburg, Sweden.

[9] Mcmillen, Tyler and Alain G. (2003) "Whip waves",Physica D 184: 192-225.

[10] Maziar A. S., and Andre S.(2015). A new model for human-like postural control in walking. Intelligent Robotics and Systems (IROS).ISBN1 5667238.DOI:10.1109/IROS.2015.7354192.

[11] Moore , Douglas B. and Stephen W. S. (1990). The Experimental Response of an Impacting Pendulum System.International Journal of nonlinear mechanics Vol.25 pp 1-16.

[12] Prichani J.S, (2010), AnM.sc. thesis, Equations of motion for n-tupple Pendulum systems, MasindeMuliro University, pg (38-39).

[13] Prichani J. S.,Sakwa T.W, Ayodo Y.K and Sarai C.A (2012). Equations of motion for multiple-pendula system. International Journal of Physics and Mathematical Sciences ISSN: 2277211vol.2(2) pp.137140

[14] Tri-Nhut D., Ran L., Chan Y., U-Xuan T., (2015).Design of an infrastructureless in-door localization device using an IMU sensor. Robotics and Biomimetics (ROBIO).ISBN 15806590. DOI: 10.1109/ROBIO.2015.7419086.

[15]Zhenglong S., Nico R. (2014).An energy efficient dynamic gait for a Nao robot.Institute of Electrical and Electronic Engineers. ISBN: NO. 14447143 DOI: 10.1109/ICARSC. 2014.6849797. pp 267-272.

\section{Author Profile}

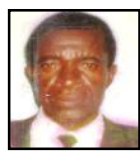

Prichani Joseck Simiyu Saratuki was born at Bungoma, County, Kenya. He holds a Bachelor of Education Science degree, and specialized in Mathematics and Physics, from Kenyatta University (KU), Nairobi, Kenya, and a Master of Science degree in Applied Mathematics from Masinde Muliro University of Science and Technology (MMUST), Kakamega, Kenya and is currently pursuing a $\mathrm{PhD}$ in applied mathematics at Jaramogi Oginga Odinga University of Science and Technology (JOOUST), Nairobi, Kenya. Affiliation: Jaramogi Oginga Odinga University of Science and Technology (MMUST), BONDO, KENYA. He is currently a part-time lecturer at Masinde Muliro University of Science and Technology-Webuye and Bungoma campuses. He has participated in many conferences including publishing four papers in refereed journals..He has much interest in the study of numerical methods and their respective applications in modeling physical phenomenon in Mathematics, sciences and engineering.

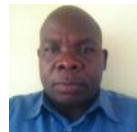

Prof. Thomas Sakwa holds a Bachelor of Science degree, Masters of Science and a PhD in Condensed Matter Physics from Moi University, Kenya. Affiliation: Masinde Muliro University of Science and Technology (MMUST), Kakamega, Kenya. He is currently a full professor of physics Professor of Physics at Masinde Muliro University of Science and Technology Department of Physics. Working experience is as follows: Graduate Assistant, Lecturer, 


\section{International Journal of Science and Research (IJSR) \\ ISSN (Online): 2319-7064}

Index Copernicus Value (2015): 78.96 | Impact Factor (2015): 6.391

Moi University - Department of Physics Kenya - Eldoret, Uasin Gishu (Dec 1994-Nov 2003). Full professor of physics at Kakamega, Kenya. He is currently a full professor of physics Professor of Physics at Masinde Muliro University of Science and Technology Department of Physics (2003 todate)He has supervised, trained and taught over 30 postgraduate students and published 31 papers in refereed journals

Prof. Naftali Omolo Ongati holds a Bachelor of Education (Science) from University of Nairobi, Kenya (1973-1976), Diploma in Mathematics curriculum (Leeds University, UK), Master of Science degree in Applied Mathematics from Odessa state University, Ukraine (1977-1979), and a PhD in applied Mathematics from University of Pretoria (1993-1997), South Africa. Affiliation: Jaramogi Oginga Odinga University of Science and Technology, (JOOUST), Bondo, Kenya. He is currently the Dean School of Mathematics and Actuarial Sciences- JOOUST. He holds the rank of Full Professor of mathematics department. He has published over 42 papers in respected journals, published 6 books and supervised over 40 postgraduate students and $18 \mathrm{PhDs}$. Teaching experience is as follows: University of Pretoria South Africa, Maseno University-Kenya, Kenyatta University-Kenya, Egerton University Kenya, Kenya Science Teachers college-Kenya and Jaramogi Oginga Odinga University of Science and Technology-Kenya. He has academic research interests in Numerical Analysis, Lie Groups and Differential Equations, and Mathematical modeling.

Volume 6 Issue 7, July 2017 www.ijsr.net 